\title{
On the Scalar Manifold of Exceptional Supergravity
}

\author{
Sergio L. Cacciatori ${ }^{1,4}$, Bianca L. Cerchiai ${ }^{2,4}$, and Alessio Marrani ${ }^{3}$ \\ 1 Dipartimento di Scienze ed Alta Tecnologia, \\ Università degli Studi dell'Insubria, Via Valleggio 11, 22100 Como, Italy \\ sergio.cacciatorieuninsubria.it \\ 2 Dipartimento di Matematica, \\ Università degli Studi di Milano, Via Saldini 50, 20133 Milano, Italy \\ bianca.cerchiai@unimi.it \\ 3 Physics Department,Theory Unit, CERN, \\ CH 1211, Geneva 23, Switzerland \\ alessio.marraniecern.ch \\ 4 INFN, Sezione di Milano \\ Via Celoria, 16, 20133 Milano, Italy
}

\begin{abstract}
We construct two parametrizations of the non compact exceptional Lie group $G=E_{7(-25)}$, based on a fibration which has the maximal compact subgroup $K=\frac{E_{6} \times U(1)}{\mathbb{Z}_{3}}$ as a fiber. It is well known that $G$ plays an important role in the $\mathcal{N}=2 d=4$ magic exceptional supergravity, where it describes the U-duality of the theory and where the symmetric space $\mathcal{M}=\frac{G}{K}$ gives the vector multiplets' scalar manifold.

First, by making use of the exponential map, we compute a realization of $\frac{G}{K}$, that is based on the $E_{6}$ invariant $d$-tensor, and hence exhibits the maximal possible manifest $\left[\left(E_{6} \times U(1)\right) / \mathbb{Z}_{3}\right]$-covariance. This provides a basis for the corresponding supergravity theory, which is the analogue of the Calabi-Vesentini coordinates.

Then we study the Iwasawa decomposition. Its main feature is that it is $S O(8)$-covariant and therefore it highlights the role of triality. Along the way we analyze the relevant chain of maximal embeddings which leads to $S O(8)$.

It is worth noticing that being based on the properties of a "mixed" Freudenthal-Tits magic square, the whole procedure can be generalized to a broader class of groups of type $E_{7}$.
\end{abstract}

Talk given at the XVII European Workshop on String Theory, held at the University of Padua, September 5-9, 2011 


\section{The "mixed" magic square and the 56 of the Lie algebra $\mathfrak{e}_{7(-25)}$}

Exceptional Lie groups act as symmetries in many physical systems. In particular, non compact forms of the group $E_{7}$ enter as U-duality of $d=3$ and $d=4$ supergravity theories. Here we focus on the $\mathcal{N}=2 d=4$ magic exceptional supergravity, where the relevant real form is $G=E_{7(-25)}$.

As the first step we need to construct the Lie algebra $\mathfrak{e}_{7(-25)}$. To this aim, we are going to follow the technique outlined in Sec. 7 of [1], which is based on the non-symmetric "mixed" magic square [2, 3, 4]:

Table 1: The "mixed" magic square

\begin{tabular}{|c|c|c|c|c|}
\hline & $\mathbb{R}$ & $\mathbb{C}$ & $\mathbb{H}$ & $\mathbb{O}$ \\
\hline $\mathbb{R}$ & $S O(3)$ & $S U(3)$ & $U S p(6)$ & $F_{4(-52)}$ \\
\hline $\mathbb{C}$ & $S U(3)$ & $S U(3) \oplus S U(3)$ & $S U(6)$ & $E_{6(-78)}$ \\
\hline $\mathbb{H}_{S}$ & $S p(6, \mathbb{R})$ & $S U(3,3)$ & $S O^{*}(12)$ & $E_{7(-25)}$ \\
\hline $\mathbb{O}_{S}$ & $F_{4(4)}$ & $E_{6(2)}$ & $E_{7(-5)}$ & $E_{8(-24)}$ \\
\hline
\end{tabular}

The rows and the columns contain the division algebras of the real numbers $\mathbb{R}$, the complex numbers $\mathbb{C}$, the quaternions $\mathbb{H}$, the octonions $\mathbb{O}$ and their split forms $\mathbb{H}_{S}$ and $\mathbb{O}_{S}$.

Then the Tits formula gives the Lie algebra $\mathcal{L}$ corresponding to row $\mathbb{A}$ and column $\mathbb{B}$ as $[4]$ :

$$
\mathcal{L}(\mathbb{A}, \mathbb{B})=\operatorname{Der}(\mathbb{A}) \oplus \operatorname{Der}\left(\mathfrak{J}_{3}(\mathbb{B})\right) \dot{+}\left(\mathbb{A}^{\prime} \otimes \mathfrak{J}_{3}^{\prime}(\mathbb{B})\right) .
$$

Here, the symbol $\oplus$ denotes direct sum of algebras, whereas $\dot{+}$ stands for direct sum of vector spaces. Furthermore, Der means the linear derivations, $\mathfrak{J}_{3}(\mathbb{B})$ denotes the rank-3 Jordan algebra on $\mathbb{B}$, and the priming amounts to considering only traceless elements. One of the main ingredients entering in the last term is the Lie product, which extends the multiplication to $\mathbb{A}^{\prime} \otimes \mathfrak{J}_{3}^{\prime}(\mathbb{B})$. Its explicit expression for $\mathbb{A}=\mathbb{H}_{S}$ and $\mathbb{B}=\mathbb{O}$ can be found e.g. in [5].

For the Lie algebra of $E_{7(-25)}$ the Tits formula (1) yields:

$$
\mathfrak{e}_{7(-25)}=\mathcal{L}\left(\mathbb{H}_{S}, \mathbb{O}\right)=\operatorname{Der}\left(\mathbb{H}_{S}\right) \oplus \operatorname{Der}\left(\mathfrak{J}_{3}(\mathbb{O})\right) \dot{+}\left(\mathbb{H}_{S}^{\prime} \otimes \mathfrak{J}_{3}^{\prime}(\mathbb{O})\right)=\mathfrak{s l}(2, \mathbb{R}) \oplus \mathfrak{f}_{4} \dot{+}\left(\mathbb{H}_{S}^{\prime} \otimes \mathfrak{J}_{3}^{\prime}(\mathbb{O})\right)
$$

The second step is to identify the subalgebra $\mathfrak{K}$ generating the maximal compact subgroup $K:=\left(E_{6(-78)} \times U(1)\right) / \mathbb{Z}_{3}$ of $E_{7(-25)}$. This can be achieved by using the Tits formula (1) once more to compute the manifestly $\mathfrak{f}_{4}$-covariant expression for $\mathfrak{e}_{6(-78)}$ :

$$
\mathfrak{e}_{6(-78)}=\mathcal{L}(\mathbb{C}, \mathbb{O})=\mathcal{L}(\mathbb{R}, \mathbb{O}) \dot{+}\left(i \otimes \mathfrak{J}_{3}^{\prime}(\mathbb{O})\right)=\operatorname{Der}\left(\mathfrak{J}_{3}(\mathbb{O})\right) \dot{+}\left(i \otimes \mathfrak{J}_{3}^{\prime}(\mathbb{O})\right)=\mathfrak{f}_{4} \dot{+}\left(i \otimes \mathfrak{J}_{3}^{\prime}(\mathbb{O})\right),
$$

where we are picking the only imaginary unit $i \in \mathbb{H}_{S}$ which satisfies $i^{2}=-1$. Thus, we obtain:

$$
\mathfrak{K}=\operatorname{ad}_{i} \oplus \operatorname{Der}\left(\mathfrak{J}_{3}(\mathbb{O})\right) \dot{+}\left(i \otimes \mathfrak{J}_{3}^{\prime}(\mathbb{O})\right) \text {, }
$$

with $a d_{i} \in \mathbb{H}_{S}$ the adjoint action of $i$, generating the maximal compact subgroup $U(1)$ of the group $S L(2, \mathbb{R})$ appearing in (2).

An explicit construction of the matrices $\phi_{I}, I=1, \ldots, 78$, realizing the $\mathfrak{e}_{6(-78)}$ subalgebra in its irreducible representation Fund $=\mathbf{2 7}$ has been performed e.g. in Sec. 2.1 of [6] by making use of (3) and of the explict expression of $\mathfrak{f}_{4(-52)}$ in its irrep. Fund $=\mathbf{2 6}$ previously computed in [7].

Finally, by putting together all these ingredients, we find that an explicit symplectic realization of the Lie algebra $\mathfrak{e}_{7(-25)}$ in its irreducible representation Fund $=\mathbf{5 6}$ is as follows [8].

The generators of the maximal compact subgroup $K$ (antihermitian matrices):

$$
\mathfrak{e}_{6(-78)}: \quad Y_{I}=\left(\begin{array}{c|c|c|c}
\phi_{I} & \overrightarrow{0}_{27} & 0_{27} & \overrightarrow{0}_{27} \\
\hline \overrightarrow{0}_{27}^{T} & 0 & \overrightarrow{0}_{27}^{T} & 0 \\
\hline 0_{27} & \overrightarrow{0}_{27} & -\phi_{I}^{T} & \overrightarrow{0}_{27} \\
\hline \overrightarrow{0}_{27}^{T} & 0 & \overrightarrow{0}_{27}^{T} & 0
\end{array}\right), I=1, \ldots, 78 ;
$$




$$
\mathfrak{u}(1): \quad Y_{79}=\left(\begin{array}{c|c|c|c}
\frac{i}{\sqrt{6}} I_{27} & \overrightarrow{0}_{27} & 0_{27} & \overrightarrow{0}_{27} \\
\hline \overrightarrow{0}_{27}^{T} & -i \sqrt{\frac{3}{2}} & \overrightarrow{0}_{27}^{T} & 0 \\
\hline 0_{27} & \overrightarrow{0}_{27} & -\frac{i}{\sqrt{6}} I_{27} & \overrightarrow{0}_{27} \\
\hline \overrightarrow{0}_{27}^{T} & 0 & \overrightarrow{0}_{27}^{T} & i \sqrt{\frac{3}{2}}
\end{array}\right) ;
$$

The generators of the coset $\mathcal{M}=G / K$ (hermitian matrices):

$$
\begin{array}{rl|c|c|c}
Y_{\alpha+79}= & \frac{1}{2}\left(\begin{array}{c|c|c|c}
0_{27} & \overrightarrow{0}_{27} & 2 i A_{\alpha} & i \sqrt{2} \vec{e}_{\alpha} \\
\hline \overrightarrow{0}_{27}^{T} & 0 & i \sqrt{2} \vec{e}_{\alpha}^{T} & 0 \\
\hline-2 i A_{\alpha} & -i \sqrt{2} \vec{e}_{\alpha} & 0_{27} & \overrightarrow{0}_{27} \\
\hline-i \sqrt{2} \vec{e}_{\alpha}^{T} & 0 & \overrightarrow{0}_{27}^{T} & 0
\end{array}\right), \alpha=1, \ldots, 27 ; \\
Y_{\alpha+106} & =\frac{1}{2}\left(\begin{array}{c|c|c|c}
0_{27} & \overrightarrow{0}_{27} & -2 A_{\alpha} & \sqrt{2} \vec{e}_{\alpha} \\
\hline \overrightarrow{0}_{27}^{T} & 0 & \sqrt{2} \vec{e}_{\alpha}^{T} & 0 \\
\hline-2 A_{\alpha} & \sqrt{2} \vec{e}_{\alpha} & 0_{27} & \overrightarrow{0}_{27} \\
\hline \sqrt{2} \vec{e}_{\alpha}^{T} & 0 & \overrightarrow{0}_{27}^{T} & 0
\end{array}\right), \alpha=1, \ldots, 27 .
\end{array}
$$

Here $I_{n}$ is the $n \times n$ identity matrix, $0_{27}$ is the $27 \times 27$ null matrix, $\overrightarrow{0}_{n}$ is the zero vector in $\mathbb{R}^{n}$, and $\vec{e}_{\alpha}, \alpha=1, \ldots, 27$, is the canonical basis of $\mathbb{R}^{27}$.

The matrices $A_{\alpha}$ are defined in terms of the $d$-tensor of the 27 of $E_{6(-78)}$. There is a cubic form, which is defined for any $j_{1}, j_{2}, j_{3} \in \mathfrak{J}_{3}(\mathbb{O})$ as [9, 10, 11]:

$$
\operatorname{Det}\left(j_{1}, j_{2}, j_{3}\right):=\frac{1}{3} \operatorname{Tr}\left(j_{1} \circ j_{2} \circ j_{3}\right)+\frac{1}{6} \operatorname{Tr}\left(j_{1}\right) \operatorname{Tr}\left(j_{2}\right) \operatorname{Tr}\left(j_{3}\right)-\frac{1}{6}\left(\operatorname{Tr}\left(j_{1}\right) \operatorname{Tr}\left(j_{2} \circ j_{3}\right)+\text { cyclic perm. }\right),(9)
$$

where $\circ$ is the product in $\mathfrak{J}_{3}(\mathbb{O})$. By choosing a basis $\left\{j_{a}\right\}_{a=1, \ldots, 26}$ of $\mathfrak{J}_{3}^{\prime}(\mathbb{O})$ normalized as $\left\langle j_{a}, j_{b}\right\rangle:=$ $\operatorname{Tr}\left(j_{a} \circ j_{b}\right)=2 \delta_{a b}$, a completion to a basis for $\mathfrak{J}_{3}(\mathbb{O})$ can be obtained by adding $j_{27}=\sqrt{\frac{2}{3}} I_{3}$. Then the matrices $A_{\alpha}$ 's are $27 \times 27$ symmetric matrices, whose components, explicitly computed in [5], satisfy the following relation [9]:

$$
\left(A_{\alpha}\right)_{\gamma}^{\beta}=\frac{3}{2} \operatorname{Det}\left(j_{\alpha}, j_{\gamma}, j_{\beta}\right)=: \frac{1}{\sqrt{2}} d_{\alpha \gamma \beta},
$$

where $d_{\alpha \gamma \beta}=d_{(\alpha \gamma \beta)}$ is the totally symmetric rank-3 invariant $d$-tensor of the $\mathbf{2 7}$ of of $E_{6(-78)}$, with a normalization suitable to match $\operatorname{Det}\left(j_{\alpha}, j_{\gamma}, j_{\beta}\right)$ given by $(9)$. Whenever the choice of the basis $\left\{j_{\alpha}\right\}$ is exploited in order to distinguish the identity matrix from the traceless ones, the $d_{\alpha \beta \gamma}$ of $E_{6}$ has a maximal manifestly $F_{4(-52)}$-invariance only. However, it is crucial to point out that, being expressed only in terms of the invariant $d$-tensor, the result (10) does not depend on the particular choice of the basis $\left\{j_{\alpha}\right\}$. Thus, the expressions of $Y_{\alpha+79}(7)$ and of $Y_{\alpha+106}(8)$ exhibit the maximal manifest compact $\left[\left(E_{6} \times U(1)\right) / \mathbb{Z}_{3}\right]$ covariance.

A couple of remarks on the properties of the matrices $Y_{A}$ 's are in order. The first is that they satisify:

$$
Y_{A} \in \mathfrak{u s p}(28,28), A=1, \ldots, 133 .
$$

Moreover, in order to guarantee that the period of the maximal torus in the $E_{6}$ subgroup equals $4 \pi$, the standard choice for the period of the spin representations of the orthogonal subgroups [7, 6], the matrices 
$Y_{A}$ 's are orthonormalized as $\left\langle Y, Y^{\prime}\right\rangle_{\mathbf{5 6}}:=\frac{1}{12} \operatorname{Tr}\left(Y Y^{\prime}\right)$ with signature $\left(-{ }^{79},+{ }^{54}\right)$. As a consequence, the components $\left(A_{\alpha}\right)_{\gamma}^{\beta}:=A_{\alpha \beta \gamma}$ are normalized as $A_{\alpha \beta \gamma} A^{\eta \beta \gamma}=5 \delta_{\alpha}^{\eta}$.

This is consistent with the normalization of the $d$-tensor (of $E_{6(-26)}$ ) adopted e.g. in [12], which is dictated by the expression $f(z):=\frac{1}{3 !} d_{\alpha \beta \gamma} z^{\alpha} z^{\beta} z^{\gamma}$ for the Kähler-invariant $\left(\left(X^{0}\right)^{2}\right.$-rescaled) holomorphic prepotential function characterizing special Kähler geometry (see e.g. [13, 14, 15], and Refs. therein).

\section{Manifestly $\left[\left(E_{6} \times U(1)\right) / \mathbb{Z}_{3}\right]$-covariant Construction of the Coset $\mathcal{M}$}

In this Section we construct a manifestly $\left[\left(E_{6} \times U(1)\right) / \mathbb{Z}_{3}\right]$-covariant parametrization of the symmetric space $\mathcal{M}=\frac{E_{7(-25)}}{\left(E_{6(-78)} \times U(1)\right) / \mathbb{Z}_{3}}$. As we have seen in the previous Sec. 11 it is generated by the matrices $Y_{79+I}$, (7) and (8) with $I=1, \ldots, 54$. Through the exponential mapping, it can be defined as follows:

$$
\mathcal{M}:=\exp \left(\sum_{\alpha=1}^{27} x_{\alpha} Y_{106+\alpha}+y_{\alpha} Y_{79+\alpha}\right), \text { with } x_{\alpha} \in \mathbb{R}, y_{\alpha} \in \mathbb{R}, \text { for } \alpha=1, \ldots, 27 .
$$

In order to make the complex structure of $\mathcal{M}$ manifest, it is convenient to introduce the following complex linear combinations of the matrices:

$$
\zeta_{\alpha}:=\frac{1}{\sqrt{2}}\left(Y_{79+\alpha}+i Y_{106+\alpha}\right), \quad \bar{\zeta}_{\alpha}:=\frac{1}{\sqrt{2}}\left(Y_{79+\alpha}-i Y_{106+\alpha}\right)
$$

together with the corresponding complex linear combinations of the parameters:

$$
z_{\alpha}:=\frac{1}{\sqrt{2}}\left(y_{\alpha}+i x_{\alpha}\right), \quad \bar{z}_{\alpha}:=\frac{1}{\sqrt{2}}\left(y_{\alpha}-i x_{\alpha}\right),
$$

which allows to rewrite $(12$ ) as

$$
\mathcal{M}:=\exp \left(\sum_{\alpha=1}^{27} \bar{z}_{\alpha} \zeta_{\alpha}+z_{\alpha} \bar{\zeta}_{\alpha}\right)
$$

By introducing the 27 dimensional complex vector $z:=\sum_{\alpha=1}^{27} z_{\alpha} \vec{e}_{\alpha}$, describing the scalar fields, and the $28 \times$ 28 matrix $\mathcal{A}:=\left(\begin{array}{c|c}-\sqrt{2} \sum_{\alpha=1}^{27} \bar{z}_{\alpha} A_{\alpha} & z \\ \hline z^{T} & 0\end{array}\right)$, the expression for $\mathcal{M}(15)$ enjoys the simple form:

$$
\mathcal{M}:=\exp \left(\begin{array}{c|c}
0 & \mathcal{A} \\
\hline \mathcal{A}^{\dagger} & 0
\end{array}\right)=\left(\begin{array}{c|c}
\operatorname{Ch}\left(\sqrt{\mathcal{A} \mathcal{A}^{\dagger}}\right) & \mathcal{A} \frac{\operatorname{Sh}\left(\sqrt{\mathcal{A}^{\dagger} \mathcal{A}}\right)}{\sqrt{\mathcal{A}^{\dagger} \mathcal{A}}} \\
\hline \frac{\operatorname{Sh}\left(\sqrt{\mathcal{A} \mathcal{A}^{\dagger}}\right)}{\sqrt{\mathcal{A} \mathcal{A}^{\dagger}}} \mathcal{A}^{\dagger} & \operatorname{Ch}\left(\sqrt{\mathcal{A}^{\dagger} \mathcal{A}}\right)
\end{array}\right) .
$$

This is a Hermitian matrix, of the same form as the finite coset representative worked out [16] for the split (i.e. maximally non-compact) counterpart $\mathcal{M}_{\mathcal{N}=8}=\frac{E_{7(7)}}{S U(8) / \mathbb{Z}_{2}}$, which is the scalar manifold of maximal $\mathcal{N}=8, D=4$ supergravity, associated to $\mathfrak{J}_{3}\left(\mathbb{O}_{S}\right)$. However, while $\mathcal{M}_{\mathcal{N}=8}$ is real, because of (11) $\mathcal{M}$ is an element of $U S p(28,28)$. 
By using the machinery of special Kähler geometry (see e.g. [13, 14, 15], and Refs. therein), the symplectic sections defining the symplectic frame associated to the coset parametrization introduced above can be directly read from (16):

$$
\mathcal{M}=:\left(\begin{array}{c|c}
u_{i}^{\Lambda}(z, \bar{z}) & v_{i \Lambda}(z, \bar{z}) \\
\hline v^{i \Lambda}(z, \bar{z}) & u_{\Lambda}^{i}(z, \bar{z})
\end{array}\right),
$$

where the symplectic index $\Lambda=0,1, \ldots 27$ (with 0 pertaining to the $\mathcal{N}=2, D=4$ graviphoton), and $i=\bar{\alpha}, 28$. Thus, the symplectic sections read (see e.g. [17, 15] and Refs. therein; subscript "28" omitted):

$$
\begin{aligned}
f_{i}^{\Lambda} \quad: \quad=\frac{1}{\sqrt{2}}(u+v)_{i}^{\Lambda}=\left(\bar{f}_{\bar{\alpha}}^{\Lambda}, f^{\Lambda}\right):=\left(\overline{\mathcal{D}}_{\bar{\alpha}} \bar{L}^{\Lambda}, L^{\Lambda}\right)=\exp \left(\frac{1}{2} K\right)\left(\overline{\mathcal{D}}_{\bar{\alpha}} \bar{X}^{\Lambda}, X^{\Lambda}\right) ; \\
h_{i \Lambda} \quad: \quad=-\frac{i}{\sqrt{2}}(u-v)_{i \Lambda}=\left(\bar{h}_{\bar{\alpha} \mid \Lambda}, h_{\Lambda}\right):=\left(\overline{\mathcal{D}}_{\bar{\alpha}} \bar{M}_{\Lambda}, M_{\Lambda}\right)=\exp \left(\frac{1}{2} K\right)\left(\overline{\mathcal{D}}_{\bar{\alpha}} \bar{F}_{\Lambda}, F_{\Lambda}\right),
\end{aligned}
$$

where $\mathcal{D}$ is the Kähler-covariant differential operator,

$$
\mathcal{V}:=\left(L^{\Lambda}, M_{\Lambda}\right)^{T}=\exp \left(\frac{1}{2} K\right)\left(X^{\Lambda}, F_{\Lambda}\right)^{T}
$$

is the symplectic vector of Kähler-covariantly holomorphic sections, and

$$
K:=-\ln \left[i\left(\bar{X}^{\Lambda} F_{\Lambda}-X^{\Lambda} \bar{F}_{\Lambda}\right)\right]
$$

is the Kähler potential determining the corresponding geometry. A more explicit expression for (16) would be needed in order to check that the prepotential $F$ does not exist (i.e., $2 F=X^{\Lambda} F_{\Lambda}=0$ [18]) in the symplectic frame we have just introduced, which can be considered the analogue of the Calabi-Vesentini basis [19, 18], whose manifest covariance is the maximal one.

\section{The Iwasawa Decomposition and the role of triality}

Now we are going to find another parametrization for the coset $\mathcal{M}$, provided by the Iwasawa decomposition. In this case the maximal manifest covariance is broken down to a subgroup $S O(8)$, thus providing a manifestly triality-symmetric description.

The manifold $\mathcal{M}$ has rank 3 , which means that the maximal dimension of the intersection between a Cartan subalgebra of $E_{7(-25)}$ and the generators of $\mathcal{M}$ is 3 . In particular, we can pick 3 such generators to be the diagonal generators of the Jordan algebra $\mathfrak{J}_{3}(\mathbb{O})$ itself, namely $h_{1}=Y_{123}, h_{2}=Y_{132}$ and $h_{3}=Y_{133}$.

The following step is to determine a basis $\mathcal{W}_{+}$of $54-3=51$ positive roots $\lambda_{i}^{+}, i=1, \ldots, 51$ with respect to $\mathfrak{H}_{3}$. Then the Iwasawa decomposition of the coset $\mathcal{M}$ is defined as:

$$
\mathcal{M}:=\exp \left(x_{1} h_{1}+x_{2} h_{2}+x_{3} h_{3}\right) \exp \left(\sum_{i=1}^{51} y_{i} \lambda_{i}^{+}\right) .
$$

As anticipated, one of its main features is that since the elements $h_{1}, h_{2}, h_{3} \in \mathfrak{h}_{3}$ commute with a 28dimensional subalgebra $\mathfrak{s o}(8)$, the Iwasawa parametrization of $\mathcal{M}$ exhibits a maximal manifest covariance given by $S O(8)$. Therefore, the 51 -dimensional linear space $\Lambda_{+}$generated by the positive roots $\mathcal{W}_{+}$is invariant under the (adjoint) action of $S O(8)$, and it decomposes into irreps. of $S O(8)$ as:

$$
\Lambda_{+}=\mathbf{1}^{3}+\mathbf{8}_{v}^{2}+\mathbf{8}_{c}^{2}+\mathbf{8}_{s}^{2}
$$

which is a manifestly triality-symmetric decomposition. In particular, at the level of algebras $\mathfrak{s o}(8)=\mathfrak{t r i}(\mathbb{O})$ with the automorphism group $\operatorname{Aut}(\mathbf{t}(\mathbb{O}))=\operatorname{Spin}(8)$ of the normed triality over the octonions $\mathbb{O}$ [20]. 
It is worth remarking that the appearance of the square for the three 8 irreps. in (23) is a consequence of the complex (special Kähler) structure of the $\operatorname{coset} \mathcal{M}$.

Moreover, it should be observed that the $S O(8)$ entering in (23) can be identified as:

$$
S O(8) \subset\left[(S O(10) \times U(1)) \cap F_{4(-52)}\right] .
$$

This can be understood by noticing that it can be obtained from both the following chains of maximal symmetric embeddings [21]:

$$
E_{7(-25)} \supset E_{6(-78)} \times U(1)^{\prime} \supset S O(10) \times U(1)^{\prime} \times U(1)^{\prime \prime} \supset S O(8) \times U(1)^{\prime} \times U(1)^{\prime \prime} \times U(1)^{\prime \prime \prime}
$$

and

$$
E_{7(-25)} \supset E_{6(-78)} \times U(1)^{\prime} \supset F_{4(-52)} \times U(1)^{\prime} \supset S O(9) \times U(1)^{\prime} \supset S O(8) \times U(1)^{\prime} .
$$

In the last line of (25) the first two $U(1)$ factors have the physical meaning of "extra" $T$-dualities generated by the Kaluza-Klein reductions, respectively $D=5 \rightarrow D=4$, and $D=6 \rightarrow D=5$.

Denoting with subscripts $U(1)$-charges, the adjoint irrep. 133 of $E_{7(-25)}$ branches according to (25) as (see e.g. [22]):

$$
\begin{aligned}
\mathbf{1 3 3}= & \mathbf{7 8}_{0}+\mathbf{1}_{0}+\mathbf{2} \mathbf{7}_{-2}+\mathbf{2 7}_{+2}^{\prime} \\
= & \mathbf{1}_{0,0}+\mathbf{1 6}_{0,-3}+\mathbf{1 6}_{0,+3}^{\prime}+\mathbf{4 5}_{0,0}+\mathbf{1}_{0,0} \\
& +\mathbf{1}_{-2,+4}+\mathbf{1 0}_{-2,-2}+\mathbf{1 6}_{-2,+1} \\
& +\mathbf{1}_{+2,-4}+\mathbf{1 0}_{+2,+2}+\mathbf{1 6}_{+2,-1}^{\prime} \\
= & \mathbf{1}_{0,0,0}+\mathbf{8}_{c, 0,-3,1}+\mathbf{8}_{s, 0,-3,-1}+\mathbf{8}_{c, 0,+3,-1}+\mathbf{8}_{s, 0,+3,+1} \\
& +\mathbf{1}_{0,0,0}+\mathbf{8}_{v, 0,0,+2}+\mathbf{8}_{v, 0,0,-2}+\mathbf{2}_{0,0,0}+\mathbf{1}_{0,0,0} \\
& +\mathbf{1}_{-2,+4,0}+\mathbf{1}_{-2,-2,+2}+\mathbf{1}_{-2,-2,-2}+\mathbf{8}_{v,-2,-2,0}+\mathbf{8}_{c,-2,+1,+1}+\mathbf{8}_{s,-2,+1,-1} \\
& +\mathbf{1}_{+2,-4,0}+\mathbf{1}_{+2,+2,-2}+\mathbf{1}_{+2,+2,+2}+\mathbf{8}_{v,+2,+2,0}+\mathbf{8}_{c,+2,-1,-1}+\mathbf{8}_{s,+2,-1,+1} .
\end{aligned}
$$

\section{Final Remarks}

It is very interesting to remark that being based only on the algebraic properties of the "mixed" FreudenthalTits magic square in Table 1 the construction of the basis with the maximal possible covariance (16) and the computation of the Iwasawa decomposition (22) described here can be both generalized [8] at least to a broader class of minimally non-compact, simple groups of type $E_{7}$ [23]. Moreover, it also turns out that, like for $\mathcal{M}$, in all these cases the maximal covariance (at least at the Lie algebra level) of the Iwasawa decomposition is given by the automorphism algebra of the corresponding normed triality [20].

\section{Acknowledgements}

The work of B.L.C. has been supported in part by the European Commission under the FP7-PEOPLE-IRG2008 Grant No. PIRG04-GA-2008-239412 String Theory and Noncommutative Geometry STRING.

\section{References}

[1] I. Yokota, Exceptional Lie Groups, arXiv:0902.0431 [math.DG] . 
[2] C. H. Barton and A. Sudbery, Magic squares and matrix models of Lie algebras, Adv. in Math. 180, 596 (2003), math/ 0203010 [math.RA].

[3] M. Günaydin, G. Sierra, P. K. Townsend, Exceptional Supergravity Theories and the Magic Square, Phys. Lett. B133B, 72 (1983).

[4] J. Tits, Algèbres alternatives, algèbres de Jordan et algèbres de Lie exceptionnelles. I. Construction, (French), Nederl. Akad. Wetensch. Proc'Ser. A 69, 223 (1966).

[5] S. L. Cacciatori, F. D. Piazza and A. Scotti, $E_{7}$ groups from octonionic magic square, arXiv:1007.4758 [math-ph].

[6] F. Bernardoni, S. L. Cacciatori, B. L. Cerchiai and A. Scotti, Mapping the geometry of the E $E_{6}$ group, J. Math. Phys. 49, 012107 (2008), arXiv:0710.0356 [math-ph].

[7] F. Bernardoni, S. L. Cacciatori, B. L. Cerchiai and A. Scotti, Mapping the geometry of the $F_{4}$ group, Adv. Theor. Math. Phys. Vol. 12, Number 4, 889 (2008), arXiv:0705.3978 [math-ph].

[8] S. L. Cacciatori, B. L. Cerchiai, A. Marrani, Magic Coset Decompositions, preprint CERN-PHTH/2012-020, arXiv:1201.6314 [hep-th].

[9] H. Freudenthal, Oktaven, Ausnahmegruppen und Oktavengeometrie, Geom. Dedicata 19, 7 (1985).

[10] L. Borsten, M. J. Duff, S. Ferrara, A. Marrani and W. Rubens, Small Orbits, arXiv: 1108.0424 [hep-th].

[11] L. Borsten, M. J. Duff, S. Ferrara, A. Marrani and W. Rubens, Explicit Orbit Classification of Reducible Jordan Algebras and Freudenthal Triple Systems, arXiv:1108.0908 [math.RA] .

[12] L. Andrianopoli, R. D’Auria, S. Ferrara and M. A. Lledó, Gauging of Flat Groups in Four Dimensional Supergravity, JHEP 0207, 010 (2002), hep-th/ 0203206.

[13] A. Strominger, Special Geometry, Commun. Math. Phys. 133, 163 (1990).

[14] B. de Wit, F. Vanderseypen and A. Van Proeyen, Symmetry Structure of Special Geometries, Nucl. Phys. B400, 463 (1993), hep-th/9210068.

[15] L. Andrianopoli, M. Bertolini, A. Ceresole, R. D’Auria, S. Ferrara, P. Fré and T. Magri, $\mathcal{N}=2 S u$ pergravity and $\mathcal{N}=2$ superYang-Mills Theory on General Scalar Manifolds : Symplectic Covariance, Gaugings and the Momentum Map, J. Geom. Phys. 23, 111 (1997), hep-th/9605032.

[16] B. de Wit and H. Nicolai, $\mathcal{N}=8$ Supergravity, Nucl. Phys. B208, 323 (1982).

[17] A. Ceresole, R. D'Auria and S. Ferrara, The Symplectic Structure of $\mathcal{N}=2$ Supergravity and its Central Extension, Nucl. Proc. Suppl. 46, 67 (1996), hep-th/9509160.

[18] A. Ceresole, R. D’Auria, S. Ferrara and A. Van Proeyen, Duality Transformations in Supersymmetric Yang-Mills Theories coupled to Supergravity, Nucl. Phys. B444, 92 (1995), hep-th/9502072.

[19] E. Calabi and E. Vesentini, On Compact, Locally Symmetric Kähler Manifolds, Ann. Math. 71, 472 (1960).

[20] J. C. Baez, The Octonions, Bull. Am. Math. Soc. 39, 145 (2002), math/ 0105155 [math-ra].

[21] R. Gilmore : “Lie Groups, Lie Algebras, and Some of Their Applications”, Dover, New York, 2006.

[22] R. Slansky, Group Theory for Unified Model Building, Phys. Rept. 79, 1 (1981).

[23] R. B. Brown, Groups of Type E $E_{7}$, J. Reine Angew. Math. 236, 79 (1969). 